\title{
Equilibrium moisture content of a crosslinked epoxy network via molecular dynamics simulations
}

\author{
M.T. Stoffels ${ }^{1}$, M.P. Staiger ${ }^{1,2}$, C.M. Bishop ${ }^{1}$ \\ ${ }^{1}$ Department of Mechanical Engineering, University of Canterbury, Private Bag \\ 4800, Christchurch 8140, New Zealand \\ 2 The MacDiarmid Institute for Advanced Materials and Nanotechnology, P.O Box \\ 600, Kelburn, Wellington 6140, New Zealand \\ E-mail: mark.stoffels@pg.canterbury.ac.nz
}

March 2016

\begin{abstract}
This study presents molecular dynamics (MD) simulation methods for determining the solubility limit of water in a crosslinked epoxy network. Procedures are first presented for dynamically crosslinking an epoxy network consisting of diglycidyl ether bisphenol A (DGEBA) and isophorone diamine (IPD). Water molecules are then introduced into the crosslinked DGEBA-IPD structure. The excess chemical potential for the absorbed water was determined through combining thermodynamic integration and Widom's test particle insertion methods. The limiting moisture uptake of the epoxy structure was determined through comparing the reduced chemical potential of the water held within the epoxy to that of pure water. The DGEBA-IPD epoxy system was found to have a moisture solubility of 3.50-3.75 wt.\% when immersed in water at $300 \mathrm{~K}$.
\end{abstract}

Keywords: Epoxy, Molecular Dynamics Simulations, Water, Solubility Limit, Excess Chemical Potential

\section{Introduction}

Thermoset epoxy polymers find many applications throughout industry due to their high chemical and heat resistance, excellent mechanical and electrical insulating properties, and dimensional stability. However, the thermomechanical properties of epoxy resins are known to be strongly influenced by environmental factors. Experimentally, these issues have been studied extensively [1-4], providing several hypotheses about the interactions between water and epoxy resins. Most commonly, results indicate that the properties of the system degrade due to plasticization in the resin caused by the presence of water.

It has been demonstrated that molecular dynamics (MD) simulations provide a powerful set of tools for characterizing polymer structures [5-8]. Several studies have utilized MD simulations to investigate the effects of moisture on cross-linked polymer systems [9-13]. Xin and Han [9] carried out an MD simulation in order to study moisture diffusivity in cross-linked epoxy networks; their study accounted for the 
influence of temperature, water concentration, and crossed-linked polymer conversion. It was shown that diffusivity increases with temperature and concentration, whereas the diffusivity decreases with an increase in polymer cross-link conversion. Wu and $\mathrm{Xu}[10,11]$ investigated the influence of water on the structure and properties of crosslinked polymer systems; in particular the density, diffusivity, fractional free volume, and polymer chain mobility were investigated.

The presence of water in a polymeric system leads to a host of damaging consequences such as degradation of mechanical strength and modulus [14, 15] hygroscopic swelling [16-18] and the accompanying mismatched swelling stresses, reduction of dielectric strength [19,20], and increase in relative permittivity [21-24]. The latter is detrimental to the performance of advanced semiconductors that use porous polymers as dielectric materials. Limiting water uptake in polymers is the key to limiting the damaging effects.

For a polymer structure there is an equilibrium mass of moisture content, the solubility limit, at which point the system absorbs no further moisture from its surroundings. Equilibrium is determined through investigations on the excess chemical potential of moisture held within the polymer and the excess chemical potential of surrounding water. Widom's test particle insertion $[25,26]$ can be used in the determination of excess chemical potentials, but becomes problematic and inefficient in highly dense systems. An accelerated Widom particle insertion method has been developed in order to calculate excess chemical potential of water help within polymer structures [27-29]. Domotor et al. [27,28] propose a grid search algorithm that identifies test particle insertion regions that would yield negligible contributions to the excess chemical potential. Hostermann et al. [29] expand this algorithm for uses with PA-6 and diglycidyl ether bisphenol A (DGEBA)-isophorone diamine (IPD).

The Gibbs ensemble provides another method of equilibrium absorption prediction. De Pablo et al. [30] combine the Gibbs ensemble method and a modified test particle grid search as a means of estimating alkane solubility in polymers. While, Kenkare et al. [31] investigate the equilibrium absorption and swelling of a polymer through a combined MD and Monte Carlo (MC) simulation study.

In this work a computational approach is developed to determine the equilibrium

moisture content of a crosslinked epoxy system through excess chemical potential methods.

\section{Fundamental Background}

Thermodynamically, the condition of chemical equilibrium over two phases, $I$ and $I I$ of species $i$ is given as,

$$
\mu_{i, I}=\mu_{i, I I}
$$


where $\mu$ is the chemical potential. Hostermann et al. [29] state that chemical equilibrium between the two states $I$ and $I I$ is additionally satisfied as follows,

$$
R T \ln \left(\frac{n_{I}}{n_{I I}}\right)=\mu_{I I}^{e x}-\mu_{I}^{e x}
$$

where $R$ is the universal gas constant, $T$ is the temperature, $n$ is the number density of the species in each state. While, $\mu^{e x}$ represents the excess chemical potential for each given state. A new term is proposed through rearranging Equation 2 such that the contributions from each state are isolated on each side of the equality. This term is referred to as the reduced chemical potential, $\tilde{\mu}$,

$$
\tilde{\mu}=\mu^{e x}+R T \ln (n)
$$

Furthermore, if chemical equilibrium exists between states $I$ and $I I$, then the following condition must also be satisfied,

$$
\tilde{\mu_{I}}=\tilde{\mu_{I}}
$$

Considering the case of a polymer submerged in pure water, the solubility of the polymer is determined through examination of Equation 4 (where, pure water is state $I$, and absorbed water is state $I I)$. At saturation, the equality will be satisfied. If $\tilde{\mu}_{I}>\tilde{\mu_{I I}}$, this indicates the system is not yet saturated, and the system will absorb further water. Conversely, if $\tilde{\mu_{I}}<\tilde{\mu_{I I}}$, this indicates the polymer is oversaturated, and there is a driving force for water to desorb. This offers the means for assessing the solubility of water in a polymeric system.

On the calculation of excess chemical potential, first consider the chemical potential of a solute $\mathrm{i}$ that is present within a polymer [32],

$$
\mu_{i}=\left.\frac{\partial A}{\partial N_{i}}\right|_{N_{p}, V, T}
$$

where $A$ is the Helmholtz free energy of the system, $V$ the volume, $T$ the temperature, and $N_{i}$ and $N_{p}$ are the number of solute and polymer molecules respectively. For an ideal gas, the Helmholtz energy is readily evaluated, as such only the excess portion of the chemical potential need be considered,

$$
\mu_{i}^{e x}=\mu_{i}-\mu_{i}^{i d e a l}=\left.\frac{\partial A^{e x}}{\partial N_{i}}\right|_{N_{p}, V, T}
$$

Widom's test particle insertion $[25,26]$ can be used in the determination of excess chemical potentials. The excess chemical potential of species $i$ in a temporarily frozen $N_{i}$-particle system is determined through observing changes in potential energy of a system with $N$ number of particles $i$, due to the insertion of an additional test particle at randomly selected positions. Widom's formulation for excess chemical potential is as follows,

$$
\mu_{i}^{e x}=-k T \ln \left\langle e^{\frac{-\Delta U_{p o t}^{(N+1 ; N)}}{k T}}\right\rangle_{N}
$$


where $k$ in the Boltzmann constant, $T$ the temperature, and $\Delta U_{p o t}^{(N+1 ; N)}$ is the change of system potential energy due to test particle insertion. The $\langle\ldots\rangle_{N}$ represents the ensemble average over the original system. However, the application of Widom's method is problematic in systems with either relatively large solute molecules, strong longrange interactions between solute and solvent species, or high density systems such as polymers. In the above instances, the insertion of a test particle results in large values of $\Delta U$, leading to a negligible contribution to $\mu_{e x}$ due to the exponential form of Equation 7.

Alternatively, thermodynamic integration methods can be used in the calculation of excess chemical potential [33-35]. A function is defined combining two states of interest as a function of a coupling parameter $\lambda$, where $0 \leq \lambda \leq 1$ and the two states of interest occur at $\lambda=0$ and $\lambda=1$. If one of the states (typically $\lambda=0$ ) describes a situation with no solute (the solute being an ideal gas e.g.), the excess chemical potential is then given as follows,

$$
\mu_{i}^{e x}=\frac{\Delta A^{e x}}{N_{i}}=\frac{1}{N_{i}} \int_{0}^{1}\left\langle\frac{\partial U_{p o t}(\lambda)}{\partial \lambda}\right\rangle d \lambda
$$

where, $N_{i}$ is the number of solute molecules, and $U_{\text {pot }}(\lambda)$ is the potential energy of the system as a function of $\lambda$. For MD, the interaction force field terms are activated as $\lambda$ increases, which directly yields changes in potential energy of the system. Limitations of thermodynamic integration method arise as singularities in the derivative of the potential energy occur while approaching the ideal gas state $(\lambda=0)$, since non-bonded interactions are fully deactivated.

Knopp et al. [36,37] describe methods for determining excess chemical potential of small molecules held within a dense microstructure; their novel methods combine thermodynamic integration methods with Widom's test particle insertion method. Individually, the Widom insertion method and thermodynamic integration are impractical for this purpose due to limitations imposed by the dense polymer structure. In approaching these issues Knopp et al. introduced an intermediate phase of water in between the real water and water as an ideal gas. This is referred to as noble water. The purpose of this intermediate state is to make the noble water molecules small enough that the Widom insertion method can be used in the calculation of the excess chemical potential. The excess chemical potential for water held within the polymer structure can be expressed by combining Equations 7 and 8 to give,

$$
\mu_{\text {water }}^{e x}=\frac{\Delta A^{e x}}{N_{w a t e r}}+\mu_{W I}^{e x}
$$

where the first term is obtained by thermodynamic integration while $\mu_{W I}^{e x}$ is found though Widom test particle insertion of the newly introduced noble water molecule.

\section{Crosslinking Procedures}

All simulations were carried out using copolymer systems consisting of diglycidyl ether bisphenol A (DGEBA) using isophorone diamine (IPD) as the curing agent. The 


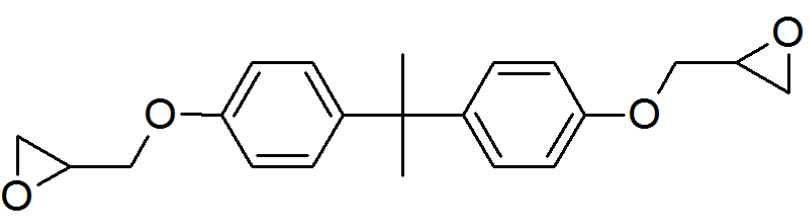

diglycidyl ether bisphenol A (DGEBA)

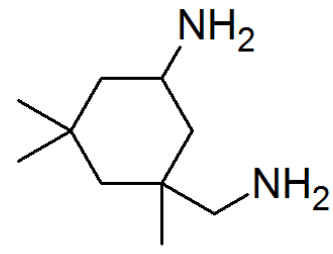

isophorone diamine (IPD)

Figure 1. Molecular structure of the resin component DGEBA (left) and curing agent IPD (right)

DGEBA
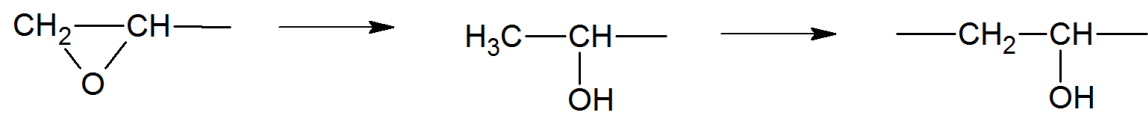

IPD:
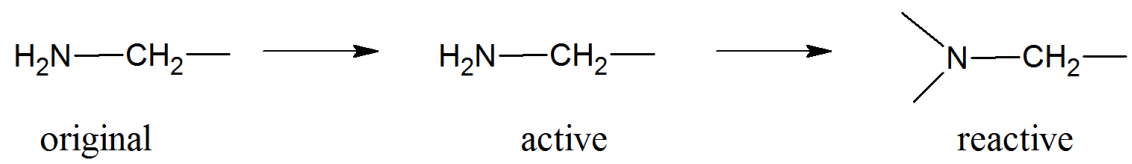

reactive

Figure 2. Activation process of end functional groups

molecular structures are presented in Figure 1. A DGEBA-IPD epoxy network was selected as it is widely used throughout industry. The crosslinking procedure presented is based upon methods developed by $\mathrm{Xu}$ et al. [6].

Initially, molecular models of DGEBA and IPD were constructed within the Avogadro molecule editor $[38,39]$. Reactive bonding sites were created by removing the active hydrogens at the end functional groups for each molecule (Figure 2). Following the creation of reactive states for DGEBA and IPD, steepest decent energy minimization was performed using Avogadro in order to geometrically optimize the structure of each molecule. Partial charges were then assigned to each atom through application of the Qeq method [40]. 256 reactive DGEBA molecules and 128 reactive IPD molecules (2:1 stoichiometric mixing ratio) were packed into $60 x 60 x 60 \AA^{3}$ simulation box using Packmol [41]. The packing strategy employed packs all molecules into specified space in such a way that the minimum distance between atoms of different molecules is greater than a fixed tolerance [42]. The tolerance was specified as $2 \AA$.

The LAMMPS (Large Scale Atomic/Molecular Massively Parallel Simulator) software package [43] was used for all molecular dynamics (MD) simulations described herein. The temperature and pressure inside the simulation box are controlled through application of the weak coupling method as presented by Berendsen et al. [44]. The CHARMM force field was employed in defining the bond, angle, and dihedral parameters [45], see Figure 3.

The potential energy contributions from bonded interactions are defined in 


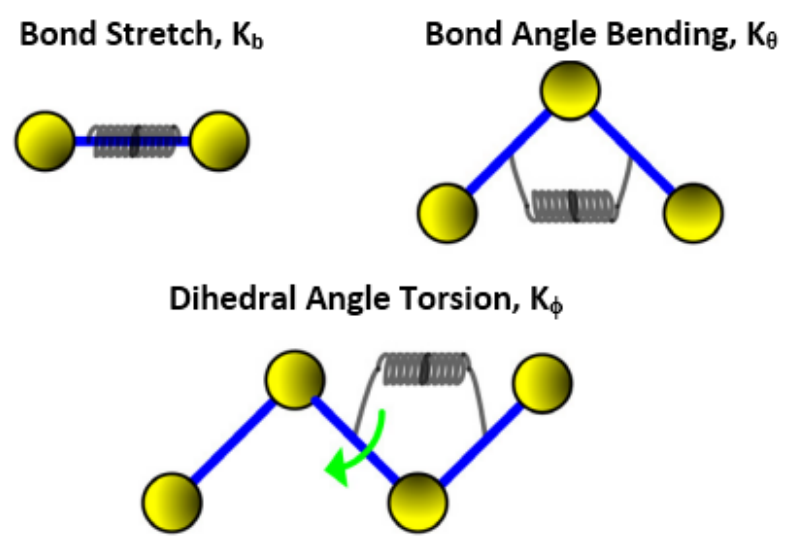

Figure 3. Illustrated terms of bonded interactions

Equation 10,

$$
E=\sum_{b} K_{b}\left(b-b_{0}\right)^{2}+\sum_{\theta} K_{\theta}\left(\theta-\theta_{0}\right)^{2}+\sum_{\phi} K_{\phi}(1+d \cos (n \phi))
$$

where $K_{b}, K_{\theta}$, and $K_{\phi}$ are force (spring) constants, $b_{0}, \theta_{0}$, and $\phi$ are equilibrium bond length, bond angle, and dihedral angle, respectively; $b$ and $\theta$ are bond length and bond angle. The CHARMM force field provides the equilibrium and force constants for each given species in the simulation. It is noted that the potential energy of the system has significant contributions from non-bonded interactions. The Lennard-Jones potential and Coulombic pairwise interactions are calculated for all particles i,j.

$$
\begin{aligned}
& E_{L J}=\sum_{\substack{i j \\
i \neq j}} 4 \epsilon_{i j}\left[\left(\frac{\sigma_{i j}}{r_{i j}}\right)^{12}-\left(\frac{\sigma_{i j}}{r_{i j}}\right)^{6}\right] \\
& E_{e l}=\sum_{\substack{i j \\
i \neq j}} \frac{C q_{i} q_{j}}{\epsilon_{o} r_{i j}}
\end{aligned}
$$

respectively, where, $\epsilon_{i j}$ defines how strongly the particles attract each other and $\sigma_{i j}$ is the cut-off distance at which the intermolecular potential between particles $i$ and $j$ is zero. $C$ is an energy-conversion constant, $q_{i}$ and $q_{j}$ are the charges for particles $i$ and $j, \epsilon_{o}$ is the permittivity of free space, and $r_{i j}$ is the distance between the particles. The CHARMM force field provides the parameters for each pair of particles in the system.

Initially, the system was subjected to 10,000 steps of energy minimization in order to relax the simulation cell. The relaxed structure was dynamically crosslinked under a constant volume and temperate (NVT) process at 300K. The crosslinking algorithm is based on the root mean square (RMS) distance between reactive $\mathrm{CH}_{2}$ groups of DGEBA and the reactive $\mathrm{N}$ atoms of the IPD. A crosslink is formed when the RMS distance between a reactive $\mathrm{CH}_{2}$ group and a reactive $\mathrm{N}$ atom is within a specified cutoff distance. If several reactive $\mathrm{CH}_{2}-\mathrm{N}$ pairs are within the specified cutoff distance then the closest such partner becomes the sole bonding partner. Once a reactive site has formed a crosslink the site becomes non-reactive and is unable to undergo further crosslinking. 
Table 1. Lennard-Jones and Coulombic parameter dependence on the coupling parameter

\begin{tabular}{|c|c|c|c|}
\hline \multirow[b]{2}{*}{ Atom } & \multirow{2}{*}{$\begin{array}{l}\text { Partial Charge } \\
q_{i}\end{array}$} & \multicolumn{2}{|c|}{$\begin{array}{c}\text { Leonard-Jones } \\
\text { interaction parameters }\end{array}$} \\
\hline & & $r_{i}$ & $\epsilon_{i}$ \\
\hline $\mathrm{O}$ & $2 \lambda q_{\text {org }}$ & $(0.8+0.2 \lambda) r_{\text {org }}$ & $\epsilon_{\text {org }}$ \\
\hline $\mathrm{H}$ & $\lambda q_{\text {org }}$ & $r_{\text {org }}$ & $\lambda^{2} \epsilon_{\text {org }}$ \\
\hline
\end{tabular}

The crosslink density of the epoxy system was defined as the ratio of the total number of crosslink bonds that were formed to that of the theoretical maximum. For example, an epoxy network that forms 16 out of a possible 32 crosslink bonds is defined as having a $50 \%$ crosslink density. For many epoxy systems, it is difficult to achieve crosslink densities much higher than $93 \%$. Each step of the crosslinking algorithm used is as follows:

1. User specified target system crosslink density (91.5\% for this work)

2. The RMS cutoff distance is increased by $0.1 \AA$

3. Crosslink bonds are formed if within the current RMS cutoff

4. 50 ps of NVT dynamics at $300 \mathrm{~K}$ were applied to the system so as to equilibrate the crosslinked system and release the strain imposed by the formation of crosslink bonds

5. Steps 2-4 are repeated until target crosslink density has been reached.

\section{Excess Chemical Potential}

Through following and expanding upon the method presented by Knopp et al. $[36,37]$ the water solubility of a polymer network can be determined by monitoring the differential excess chemical potential, $\Delta \mu_{e x}$, between the excess chemical potential of water in the polymer network and that in the pure water environment. As discussed previously, the method being considered depends upon establishing a noble water that is an intermediate state between real water and water as an ideal gas.

The noble water model is defined as follows. At $\lambda=1$, a real water model is used, with full interactions and atom size. At $\lambda=0$, a noble water model has no Coulombic interactions, Lennard-Jones interactions of the hydrogen atoms are switched off, and the oxygen atoms in all water molecules are scaled to $80 \%$ of their original LennardJones size. This radii scaling value was chosen based on efficiency optimization of the Widom insertion process. A mathematical breakdown of how the noble water model is implemented is given in Table 1, where the subscript org denotes the original, unaltered parameter as defined by the CHARMM force field.

The step-by-step procedures used in determining the excess chemical potential is as follows: 
1. For each $\lambda$, the system is equilibrated and the potential energy is obtained.

2. Through evaluation of the integral from $\lambda=0$ to 1 the difference in Helmholtz free energy is calculated (Equation 8).

3. Widom test particle insertion of a noble water molecule $(\lambda=0)$ is then carried out in order to obtain the remaining portion of the excess chemical potential (Equation 7); and

4. The excess chemical potential is determined from Equation 9.

\subsection{Pure Water System}

Initially, the case of a pure water system was considered for validation of the selected computational method since the excess chemical potential of pure water is known $\left(\mu_{e x}\right.$ $=-23.8 \mathrm{kJmol}^{-1}$ ) [46]. 50 total MD configurations of a 500 molecule pure water system are investigated, what follows are the simulation procedures for a single configuration.

The 500 water molecules were packed into $22 x 22 \times 22 \AA^{3} \quad$ simulation box using Packmol. The packing tolerance was specified as $2 \AA$. The TIP3P water model [47] was employed in defining interaction parameters $\left(q_{\text {org }}, r_{\text {org }}\right.$, and $\left.\epsilon_{\text {org }}\right)$ as well as bonding, and angle coefficients for all water molecules within the system.

A constant pressure and temperature (NPT ensemble) MD process was carried out at $1 \mathrm{~atm}$ and $300 \mathrm{~K}$ for $50 \mathrm{ps}$ in order to optimize the system density, resulting in a final system density of $0.997 \mathrm{gcm}^{-3}$. A constant volume and temperature (NVT ensemble) process was then carried out at $300 \mathrm{~K}$ for $100 \mathrm{ps}$. This allowed the molecules to move freely within the simulation box in order to reach a fully optimized molecular arrangement. The system interaction parameters were then redefined as a function of the coupling parameter $(\lambda)$ as described previously (Table 1 ).

In order to numerically determine the excess chemical potential of the system, it is first necessary to sample the potential energy of the system at various values of $\lambda$, calculate the derivates of potential energy, and then integrate over $\lambda$. Nine equidistant values for $\lambda$ between 1 and 0 were considered as integration points (i.e. $\lambda=1.0,0.905$, $0.805, \ldots, 0.105,0.0)$. Additionally, for each $\lambda$, further values are gathered at $\lambda \pm \Delta \lambda$, where $\Delta \lambda=0.005$. This allows for a more accurate representation for the local derivatives. In total, 22 seperate values of $\lambda$ are considered, yielding a set of 22 different force fields.

$500 \mathrm{ps}$ of NVT dynamics were run at a temperature of $300 \mathrm{~K}$ for each value of $\lambda$ using the optimized molecular structure obtained previously. Every 20,000 time steps a time-averaged value was calculated over the previous 20,000 steps for each energy term $\left(U_{l j}(\lambda), U_{e l}(\lambda)\right.$, and $\left.U_{n s}\right)$, allowing for simple convergence checking of each potential energy term. Figure 4 depicts how each time-averaged contribution develops as $\lambda$ is increased from 0 to 1 . The activation of pairwise interactions is seen, while the bonded, non-scaled interactions are comparatively stable throughout.

Using the time-averaged potential energy contributions the total potential energy

of the system is calculated for each given value of $\lambda$. The term $\frac{1}{N_{i}} \frac{\partial U_{p o t}(\lambda)}{\partial \lambda}$ is numerically determined using centered difference. Table 2 provides these outputs after total 


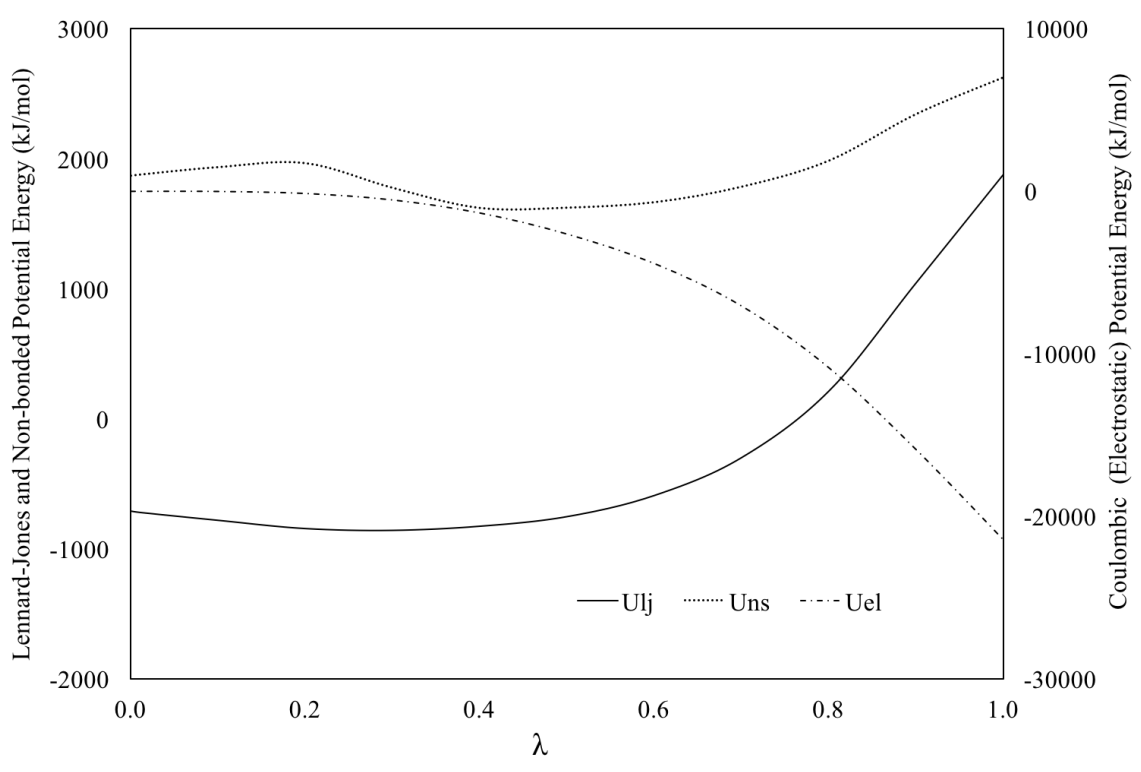

Figure 4. Individual, time-averaged energy contributions to the system potential energy as $\lambda$ in increased from 0 to 1 . The primary axis is scaled for both the Lennard-Jones (solid line) and Bonded (non-scaled) (dotted line) interactions, while the secondary axis represents the Electrostatic energy term (dash-dot line).

Table 2. Time-averaged value of $\frac{1}{N_{i}} \frac{\partial U_{\text {pot }}(\lambda)}{\partial \lambda}$ at varying MD simulation time for pure water

\begin{tabular}{rrrr}
\hline$\lambda$ & \multicolumn{1}{c}{$50 \mathrm{ps}$} & $200 \mathrm{ps}$ & $500 \mathrm{ps}$ \\
\hline 0.000 & 0.619 & 0.626 & 0.627 \\
0.105 & 0.406 & 0.411 & 0.411 \\
0.205 & -1.919 & -1.941 & -1.942 \\
0.305 & -8.062 & -8.151 & -8.155 \\
0.405 & -11.93 & -12.06 & -12.07 \\
0.505 & -16.37 & -16.55 & -16.57 \\
0.605 & -22.69 & -22.94 & -22.96 \\
0.705 & -31.31 & -31.66 & -31.68 \\
0.805 & -41.80 & -42.26 & -42.29 \\
0.905 & -52.13 & -52.70 & -52.73 \\
1.000 & -68.52 & -69.27 & -69.32 \\
\hline$\frac{1}{N_{i}} \int_{0}^{1}\left\langle\frac{\partial U_{\text {pot }}(\lambda)}{\partial \lambda}\right\rangle d \lambda$ & -21.82 & -22.06 & -22.08 \\
\hline
\end{tabular}

simulation times of 50, 200 and 500 ps. The integral over the potential energy derivative, representing the Helmholtz free energy difference of the system, is obtained by a trapezoidal integration method, the results of which are given as the final row of Table 2. The integral is observed to approach convergence with increasing MD simulation time, with values of $-21.82,-22.06$ and $-22.08 \mathrm{kJmol}^{-1}$ after simulation times of 50, 200 and 500 ps, respectively. 


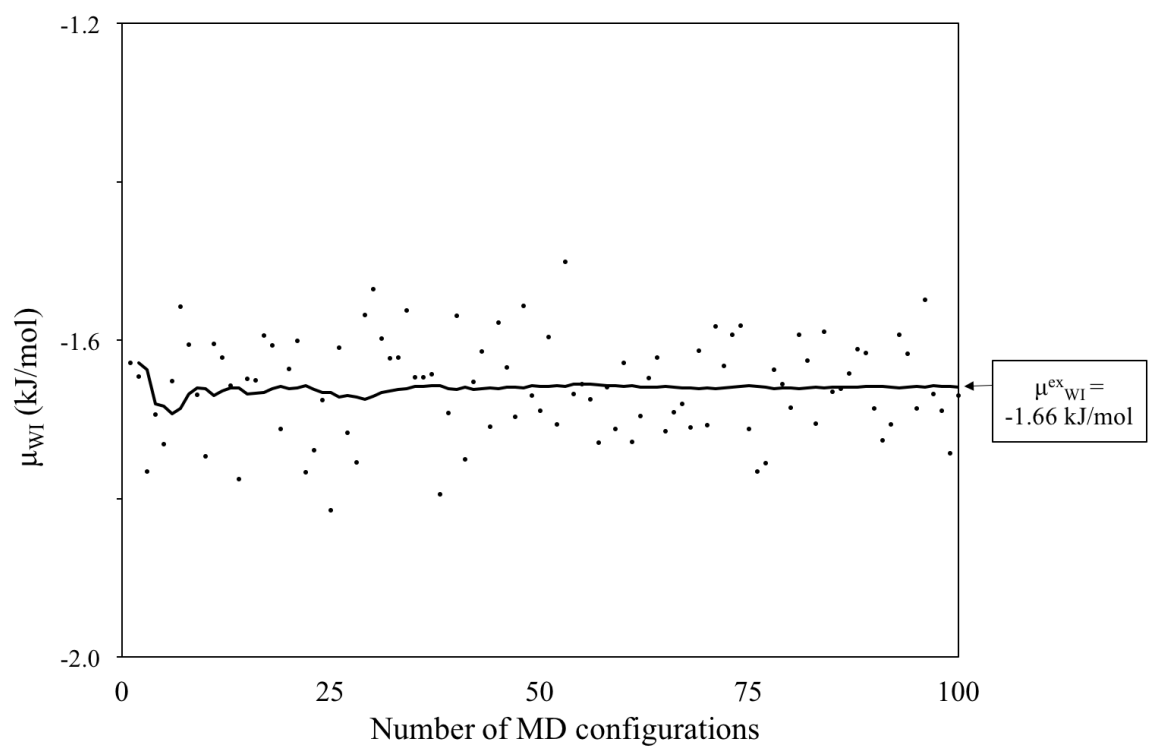

Figure 5. The excess chemical potential of noble water as calculated through Widom's test particle insertion method for 100 separate MD configurations (500-600ps). The solid line representing the moving average up to the current MD frame.

After all values of $\lambda$ have been processed through thermodynamic integration, a test particle insertion of a noble water molecule is needed to calculate the final contribution to the excess chemical potential. In order to do this, the simulation box was divided into a uniformly spaced three-dimensional grid pattern ( $25 \times 25 \times 25)$, where each grid point serves as the insertion coordinates for the test particle. The noble water molecule is placed at each point in the insertion grid for each of the 100 sampled MD configurations, each of which are sampled at 1 ps intervals from 500-600 ps during the course of the simulation. Therefore, for each of the $100 \mathrm{MD}$ configurations there are 15,625 insertions that contribute a single data point towards the calculation of $\mu_{W I}^{e x}$ using Equation 3. The resulting value of $\mu_{W I}^{e x}$ is taken as an average of all 100 configurations. Figure 5 illustrates the progress of these calculations, where each data point indicates a single application of Equation 3 to a given MD frame over the 15,625 insertion points. The excess chemical potential of the noble water is found to be $\mu_{W I}^{e x}=-1.66 \mathrm{kJmol}^{-1}$ with a standard deviation of $0.062 \mathrm{kJmol}^{-1}$.

The excess chemical potential of this configuration of the pure water system can now be fully obtained through Equation 9, wherein the excess potential found through thermodynamic integration $\left(-22.08 \mathrm{kJmol}^{-1}\right)$ is added to the value found through Widom's insertions method $\left(-1.66 \mathrm{kJmol}^{-1}\right)$, giving a total excess chemical potential of $-23.74 \mathrm{kJmol}^{-1}$.

The excess chemical potential for the pure water system is taken as an average of all 50 sampled MD configurations, see Figure 6. The excess chemical potential is found to have an average value of $-23.79 \mathrm{kJmol}^{-1}$ with a standard deviation of $0.05 \mathrm{kJmol}^{-1}$. This aligns exceptionally well with the experimentally determined value of $-23.8 \mathrm{kJmol}^{-1}$ [46]. 


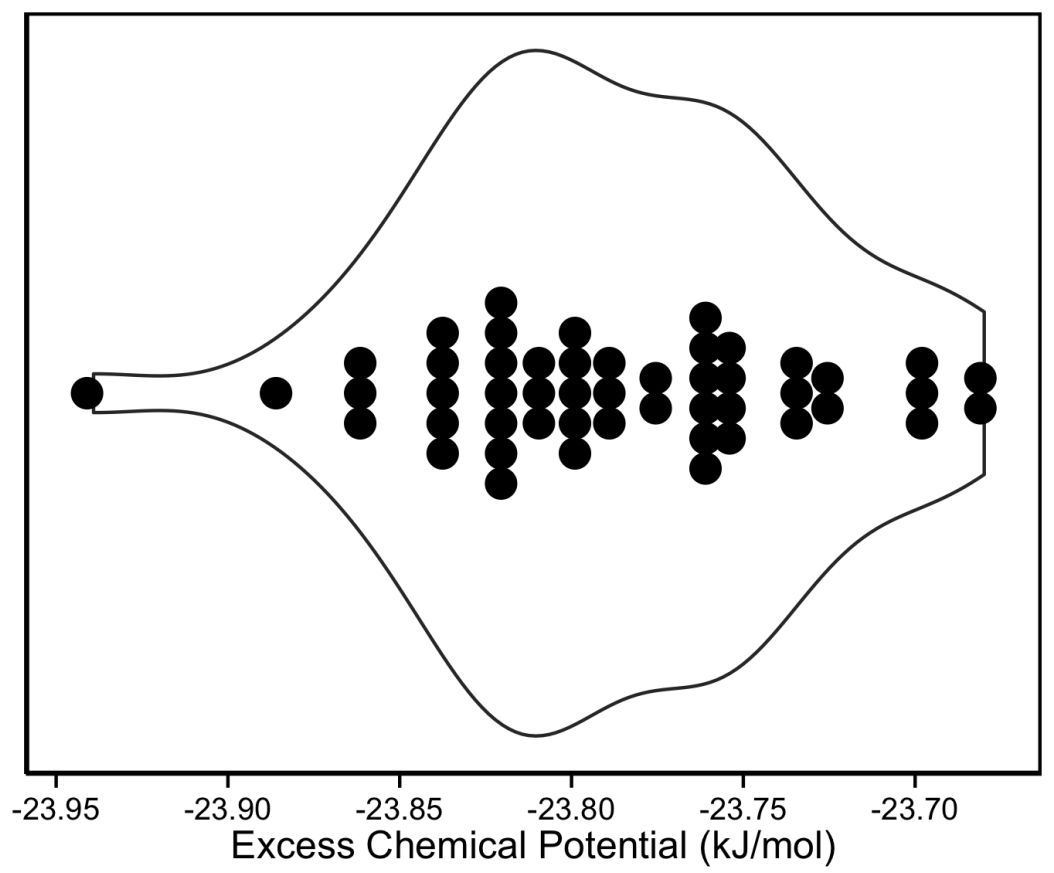

Figure 6. This plot illustrates the distribution of the excess chemical potentials calculated for each of the 50 configurations of the 500 molecule pure water system considered.

Additionally, this value shows reasonable agreement with other values found through employing different simulation techniques $\left(\mu_{W I}^{e x}=-23.4\right.$ to $\left.-24.3 \mathrm{kJmol}^{-1}\right)[48-50]$. The methods discussed have been successfully implemented in determination of the excess chemical potential of pure water, and therefore the next step is now to expand to the determination of the precise solubility of the DGEBA-IPD structure.

\subsection{Solubility Determination of DGEBA-IPD System}

At full saturation, the reduced chemical potential of absorbed water molecules held within a polymer structure will be equal to that of pure water, thereby satisfying Equation 4. For pure water, the reduced chemical potential is calculated as $\tilde{\mu}_{I}=$ $140.06 \mathrm{kJmol}^{-1}$; where $\mu_{I}^{e x}=-23.79 \mathrm{kJmol}^{-1}$, and $n_{I}=500 /$ volume of simulation cell. For each level of moisture investigated (i.e. 1\%, $2 \%, 3 \%$ etc...) the excess chemical potential is taken as an average of ten seperate DGEBA-IPD structures, each with 91.5\% crosslink density; for each structure considered, three randomly generated water molecule positions are investigated. In total, 30 values for excess chemical potential are taken for each moisture content.

Firstly, water molecules will be introduced into the crosslinked DGEBA-IPD structure at various saturation levels. The structure will then be equilibrated, and the excess chemical potential of the water molecules will be calculated using the previously 
described approach. In order to precisely determine the solubility of water in the crosslinked DGEBA-IPD system, $\Delta \mu^{e x}$ for a range of different water contents will be determined using Equation 2. Thus, it will be possible to determine at which precise water content the crosslinked DGEBA-IPD is fully saturated so as to determine the maximum solubility precisely.

What follows are the precise procedures and specific outputs used in the excess chemical potential determination for a DGEBA-IPD system saturated to a moisture content of 1 wt.\%. The number of water molecules to be inserted was based on the molecular mass of the water and epoxy. It was determined that 60 water molecules were required to obtain a moisture content of $1 \mathrm{wt} . \%$ in the system.

The 60 water molecules were then inserted at randomly assigned positions throughout the simulation box. 10,000 steps of energy minimization were performed, along with 200 ps of NVT dynamics, in order to equilibrate the saturated DGEBAIPD structure. The $\lambda$-modified force field parameters were then implemented in the simulation. NVT dynamics were run at a temperature of $300 \mathrm{~K}$ for each value of $\lambda$. Every 20,000 time steps a time-averaged value was calculated over the previous 20,000 steps for each energy term $\left(U_{l j}(\lambda), U_{e l}(\lambda)\right.$, and $\left.U_{n s}\right)$. Individually, the potential energy terms are much smaller than that of the pure water system, directly due to the fact that there are only 60 molecules in the system (whereas the pure water simulation had 500 water molecules). The same trends are seen in that the Lennard-Jones and Coulombic energy terms are activated to full-scale interactions, while the bonded (nonscaled) energy terms remain comparatively stable.

As previously, the term $\frac{1}{N_{i}} \frac{\partial U_{p o t}(\lambda)}{\partial \lambda}$ is numerically determined for each given value of $\lambda$, Table 3 provides these outputs after total simulation times of 50, 200 and 500 ps for one of the sampled polymer configurations. The integral over the potential energy derivative, representing the Helmholtz free energy difference of the system, is obtained by the trapezoidal integration method, the results of which are given as the final row of Table 3. The integral is observed to converge with increasing MD simulation time, taking values of $-19.57,-19.72$ and $-19.75 \mathrm{kJmol}^{-1}$ after simulation times of 50, 200 and 500 ps, respectively.

Widom's particle insertion of a noble water $(\lambda=0)$ molecule was then performed and $100 \mathrm{MD}$ frame collections yielded the final average value of $\mu_{W I}^{e x}=+1.53 \mathrm{kJmol}^{-1}$. By adding this to the thermodynamic integration contribution $\left(-19.75 \mathrm{kJmol}^{-1}\right)$ the final value for the excess chemical potential of the water held within the system at a 1 wt.\% moisture content was found to be $\mu_{w a t e r}^{e x}=-18.22 \mathrm{kJmol}^{-1}$.

The excess chemical potential for the $1 \%$ moisture content is taken as an average of 30 sampled MD configurations (ten DGEBA-IPD structures, each with three randomly generated water molecule positions). The excess chemical potential is found to have an average value of $-18.24 \mathrm{kJmol}^{-1}$ with a standard deviation of $0.12 \mathrm{kJmol}^{-1}$. Figure 7 shows the excess chemical potential distributions calculated for 1, 2, and 3 wt.\%.

The determination of chemical potential equilibrium, through the evaluation of equation 4 is the final step in determining the maximum solubility of water in the 
Table 3. Time-averaged value of $\frac{1}{N_{i}} \frac{\partial U_{p o t}(\lambda)}{\partial \lambda}$ at varying MD simulation time for a single system saturated to $1 \mathrm{wt} . \%$ water

\begin{tabular}{|c|c|c|c|}
\hline$\lambda$ & $50 \mathrm{ps}$ & 200ps & $500 \mathrm{ps}$ \\
\hline 0.000 & -0.624 & -0.063 & -0.063 \\
\hline 0.105 & -0.040 & -0.041 & -0.041 \\
\hline 0.205 & -0.193 & -0.195 & -0.196 \\
\hline 0.305 & -0.813 & -0.819 & -0.821 \\
\hline 0.405 & -1.203 & -1.213 & -1.215 \\
\hline 0.505 & -1.651 & -1.665 & -1.667 \\
\hline 0.605 & -2.287 & -2.307 & -2.311 \\
\hline 0.705 & -3.158 & -3.184 & -3.189 \\
\hline 0.805 & -4.216 & -4.251 & -4.258 \\
\hline 0.905 & -5.257 & -5.301 & -5.31 \\
\hline 1.000 & -6.911 & -6.968 & -6.980 \\
\hline
\end{tabular}

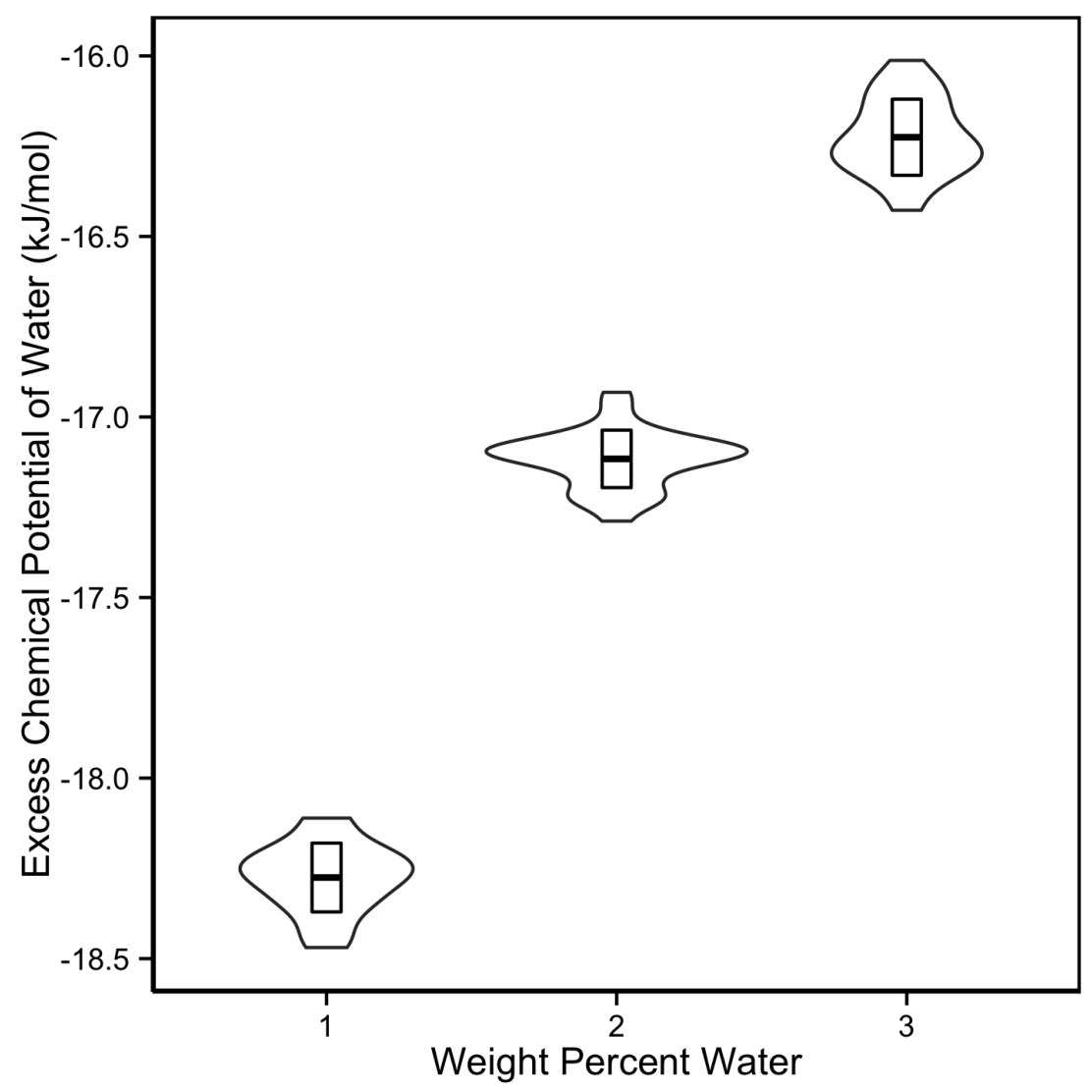

Figure 7. Each plot represents the distribution of 30 excess chemical potentials calculated for 1, 2, and 3 wt.\% water in epoxy systems. The box plots illustrate \pm one standard deviation about the mean. 


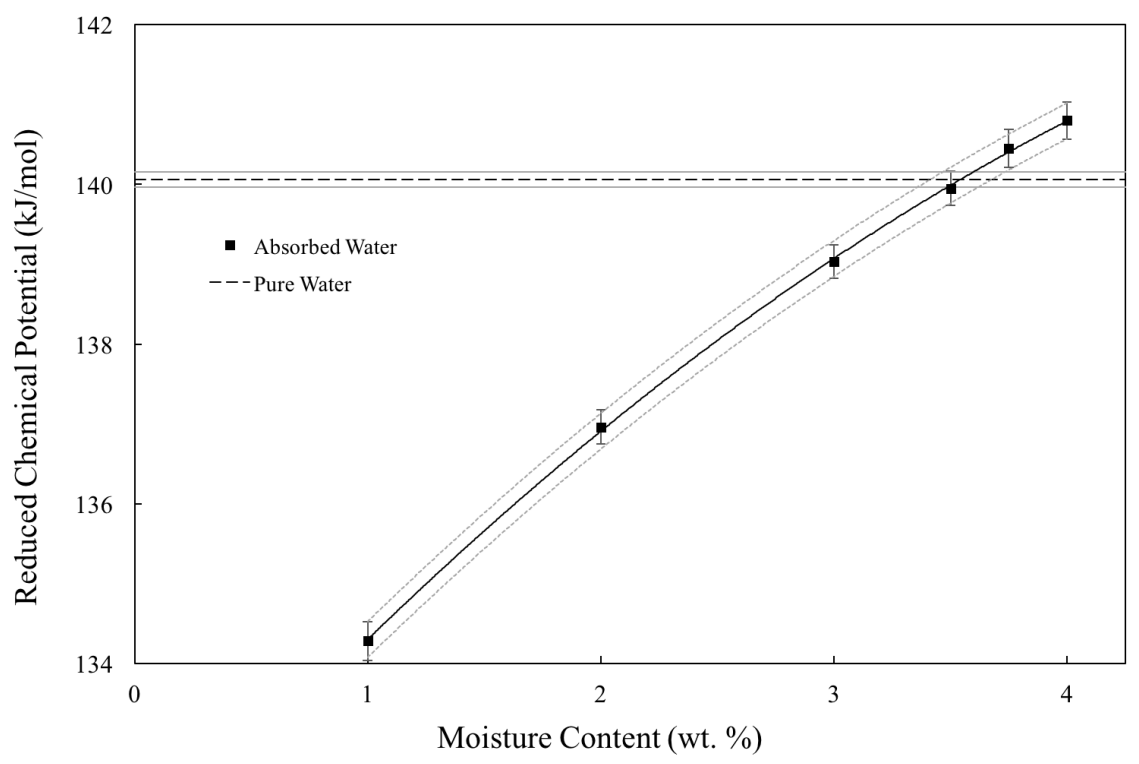

Figure 8. The reduced chemical potential, $\tilde{\mu}$, is plotted against weight content of water at $300 \mathrm{~K}, 1 \mathrm{~atm}$. Each point represents the average of 30 values found for the given weight content. The errors bars represent \pm one standard deviation from the mean. The horizontal, dashed line is a plot of the constant value $\tilde{\mu}_{\text {pure }}$, with \pm one standard deviation from the mean. Full saturation of the DGEBA-IPD system occurs at the intersection.

system. The reduced chemical potential for pure water is calculated as $\tilde{\mu}_{\text {pure }}=140.06$ $\mathrm{kJmol}^{-1}$, while for $1 \mathrm{wt} . \%$ moisture content it is calculated as $\tilde{\mu}_{\text {absorbed }}=134.28 \mathrm{kJmol}^{-1}$. Therefore, $\tilde{\mu}_{\text {pure }}>\tilde{\mu}_{\text {absorbed }}$ indicating that the DGEBA-IPD structure is not yet saturated. Additional iterations were required at higher moisture contents to obtain the maximum solubility. The calculated excess chemical potentials for each moisture content are given in Figure 8.

The DGEBA-IPD structure is found to reach full saturation in the range of 3.50-3.75 wt.\%, while published experimental values for maximum moisture content for DGEBAIPD systems are reported in the range of 1.5-2.6 wt.\% [51-53]. Marie et al. [51] report an equilibrium content of 2.3 wt.\% for a DGEBA-IPD network at $300 \mathrm{~K}, 1 \mathrm{~atm}$, though they do not provide the crosslink density for the system. Berruet et al. [52] provide a value of 2.6 wt.\% for DGEBA-IPD at $300 \mathrm{~K}, 1 \mathrm{~atm}$, the crosslink density is again not provided. Finally, Morel et al. [53] report 1.5 wt.\% at 293K, 1 atm, with no specifics provided for crosslink density. For all simulations the crosslink density used was 91.5\%, and while this value was chosen to be reasonably attainable, the experimental values may not align at the same crosslink density. Furthermore, crosslink density directly influences the resulting equilibrium moisture content, where higher crosslink densities leave fewer available polar bonding sites, hindering moisture solubility [10,51,54]. Incorporating polar bonding would introduce another layer of complexities into the simulation, and was outside the scope of this study.

The methods presented in this work rely on differentiating very small energy 
differences, where small variations can carry large impacts. Efforts have been made to minimise these kinds of errors, with the rigorous time-averaging techniques used. Further work is needed in order to gain a complete understanding of the specific interactions between epoxy and water.

\section{Conclusions}

Through application of a hybrid thermodynamic integration and Widom's test particle insertion method the precise excess chemical potential for water held within the polymer structure, and therefore the equilibrium moisture content was determined with reasonable agreement with the experimentally determined values. The solubility limit of water in DGEBA-IPD with $91.5 \%$ crosslink density at $300 \mathrm{~K}$ and 1 atmosphere is found to fall in the range $3.50-3.75 \mathrm{wt} . \%$.

This method holds advantages when compared to other computational methods used in the calculation of excess chemical potentials for solutes in dense microstructures. It is easily implemented into commonly available MD simulation packages, where it is only required to alter the force field parameters. As it is simply implemented into existing simulation routines, structural information about the system can be extracted simultaneously, further increasing computational efficiency.

This method does prove to be exceptionally sensitive to the energy potential function selected. In transferring these methods to other epoxy systems a new potential function will need to be implemented. Overall, results demonstrate that MD simulations can be used as a means to calculate equilibrium moisture content for crosslinked epoxy systems.

\section{References}

[1] Zhou J and Lucas J 1999 Polymer 40 5505-5512

[2] Pethrick R A, Hollins E A, McEwan I, MacKinnon A J, Hayward D, Cannon L A, Jenkins S D and McGrail P T 1996 Macromolecules 29 5208-5214

[3] Pethrick R A, Hollins E A, McEwan I, Pollock E A, Hayward D and Johncock P 1996 Polymer International $39275-288$

[4] Xiao G, Delamar M and Shanahan M 1997 Journal of Applied Polymer Science 65 449-458

[5] Varshney V, Patnaik S S, Roy A K and Farmer B L 2008 Macromolecules 41 6837-6842

[6] $\mathrm{Wu} \mathrm{C}$ and Xu W 2006 Polymer 47 6004-6009

[7] Yarovsky I and Evans E 2002 Polymer 43 963-969

[8] Fan H B and Yuen M M 2007 Polymer 48 2174-2178

[9] Xin D and Han Q 2013 Molecular Simulation 39 322-329

[10] Wu C and Xu W 2007 Polymer 48 5440-5448

[11] Wu C and Xu W 2007 Polymer 48 5802-5812

[12] Lin Y and Chen X 2005 Chemical Physics Letters 412 322-326

[13] Fan H B, Chan E K L, Wong Cell K Y and Yuen M M F 2006 Journal of Adhesion Science and Technology 20 1937-1947 ISSN 01694243

[14] Zafar A, Bertocco F, Schjødt-Thomsen J and Rauhe J 2012 Composites Science and Technology 72656 - 666 ISSN 0266-3538 
[15] Papanicolaou G, Kosmidou T, Vatalis A and Delides C 2006 Journal of Applied Polymer Science 99 1328-1339

[16] McKague Jr E, Reynolds J D and Halkias J E 1978 Journal of Applied Polymer Science 22 $1643-1654$

[17] Xiao G and Shanahan M 1998 Polymer 39 3253-3260

[18] Loh W, Crocombe A, Wahab M A and Ashcroft I 2005 International Journal of Adhesion and Adhesives 25 1-12

[19] De B and Karak N 2015 RSC Advances 5 35080-35088

[20] Li Y and Unsworth J 1994 IEEE Transactions on Dielectrics and Electrical Insulation 1 9-17

[21] Garcia C, Cohn J, Chesley C and Grace L 2014 Effects of moisture absorption on the dielectric properties of nanoclay-reinforced epoxy for radome applications vol 9

[22] Grace L 2015 Composite Structures 128 305-312

[23] Ivanova K, Pethrick R and Affrossman S 2002 Journal of Applied Polymer Science 84 1011-1024

[24] Joshi S, Pethrick R, Gilmore R, Yates L and Hayward D 1997 Journal of Adhesion 62 281-315

[25] Widom B 1982 Journal of Physical Chemistry 86 869-872

[26] Wldom B 1963 The Journal of Chemical Physics 39 2808-2812

[27] Domotor G and Hentschke R 2004 Macromolecular Theory and Simulations 13 506-511

[28] Domotor G and Hentschke R 2004 Journal of Physical Chemistry B 108 2413-2417

[29] Horstermann H, Hentschke R, Amkreutz M, Hoffmann M and Wirts-Rutters M 2010 Journal of Physical Chemistry B 114 17013-17024 cited By 15

[30] De Pablo J, Laso M and Suter U 1993 Macromolecules 26 6180-6183

[31] Kenkare N, Hall C and Khan S 2000 Journal of Chemical Physics 113 404-418

[32] Borgnakke C and Sonntag R E 2008 Fundamentals of Thermodynamics

[33] Kirkwood J 1935 The Journal of Chemical Physics 3 300-313

[34] King P 1993 Computer Simulation of Biomolecular Systems 2 267-314

[35] Van Gunsteren W, Beutler T, Fraternali F, King P, Mark A and Smith P 1993 Computer Simulation of Biomolecular Systems, Theoretical and Experimental Applications 2 315-348

[36] Knopp B and Suter U 1997 Macromolecules 30 6114-6119

[37] Knopp B, Suter U and Gusev A 1997 Macromolecules 30 6107-6113

[38] Avogadro: an open-source molecular builder and visualization tool. version 1.1.1

[39] Hanwell M D, Curtis D E, Lonie D C, Vandermeersch T, Zurek E and Hutchison G R 2012 Journal of Cheminformatics 417

[40] Rappe A K and Goddard W A 1991 Journal of Physical Chemistry 95 3358-3358

[41] Martinez L, Andrade R, Birgin E and Martinez J 2009 Journal of Computational Chemistry 30 $2157-2164$

[42] Martinez J M and Martinez L 2003 Journal of Computational Chemistry 24 819-825

[43] Plimpton S 1995 Journal of Computational Physics 117 1-19

[44] Berendsen H, Postma J, Van Gunsteren W, Dinola A and Haak J 1984 The Journal of Chemical Physics 81 3684-3690

[45] Brooks B, Bruccoleri R, Olafson B, States D, Swaminathan S and Karplus M 1983 J. Comput. Chem. 4 187-217

[46] Sarkisov G, Dashevsky V and Malenkov G 1974 Mol. Phys. 27

[47] Jorgensen W, Chandrasekhar J, Madura J, Impey R and Klein M 1983 The Journal of Chemical Physics 79 926-935

[48] Hermans J, Pathiaseril A and Anderson A 1988 Journal of the American Chemical Society 110 $5982-5986$

[49] Jorgensen W, Blake J and Buckner J 1989 Chemical Physics 129 193-200

[50] Watanabe M and Reinhardt W 1990 Physical Review Letters 65 3301-3304

[51] Chrysanthos M 2013 Novel biobased epoxy networks derived from renewable resources : Structureproperty relationships Master's thesis

[52] Berruet R, Vinard E, Calle A, Tighzert H, Chabert B, Magloire H and Eloy R 1987 Biomaterials 
$8162-171$

[53] Morel E, Bellenger V and Verdu J 1985 Polymer 26 1719-1724

[54] Soles C L and Yee A F 2000 Journal of Polymer Science, Part B: Polymer Physics 38 $792-802$ 